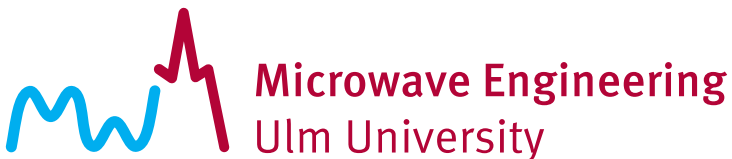

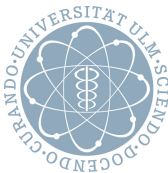

\section{Range Migration Compensation for Chirp-Sequence based Radar}

Fabian Roos, Daniel Ellenrieder, Nils Appenrodt, Jürgen Dickmann, and Christian Waldschmidt

(C) 2016 IEEE. Personal use of this material is permitted. Permission from IEEE must be obtained for all other uses, in any current or future media, including reprinting/republishing this material for advertising or promotional purposes, creating new collective works, for resale or redistribution to servers or lists, or reuse of any copyrighted component of this work in other works.

DOI: 10.1109/GEMIC.2016.7461620 


\title{
Range Migration Compensation for Chirp-Sequence based Radar
}

\author{
Fabian Roos*, Daniel Ellenrieder*, Nils Appenrodt ${ }^{\dagger}$, Jürgen Dickmann ${ }^{\dagger}$, and Christian Waldschmidt* \\ ${ }^{*}$ Institute of Microwave Engineering, Ulm University, 89081 Ulm, Germany \\ ${ }^{\dagger}$ Daimler AG, Group Research and Advanced Engineering, 89081 Ulm, Germany \\ Email: fabian.roos@uni-ulm.de
}

\begin{abstract}
To improve the range and Doppler resolution the bandwidth and the observation time need to be increased. This leads to a range migration effect and thus to a declining separability of targets. This paper compares three different modulation formats to cope with the range migration. The standard chirpsequence modulation, the bandwidth variation modulation known from literature, and the proposed chirp duration variation are analysed. A simulation is used to validate the modulation format.
\end{abstract}

\section{INTRODUCTION}

To operate driver assistance systems a precise environment perception is crucial. Especially for vulnerable road users the resolution requirements rise as presented in [1]. Only with high-resolution radar sensors such road users can be detected. Increasing the bandwidth to enhance the range resolution and transmitting a higher number of frequency ramps to improve the velocity resolution-with the drawback of an extended measurement time-will fulfill the aforementioned requirements. This results in a noticeable range cell migration effect which should be considered.

To compensate such an effect the authors of [2] are altering the bandwidth of the transmitted chirp-sequence modulation. Using such an approach for automotive parametrised radar sensors leads to a large variation of bandwidth resulting in a loss of range resolution for the first ramps. To cope with that issue an alternative modulation format is proposed.

\section{Mathematical Model: Distance Error}

The chirp-sequence $(C S)$ principle is based upon the Frequency Modulated Continuous Wave $(F M C W)$ modulation, however, the duration $T_{\mathrm{c}}$ of a single frequency ramp is shorter and several ramps are used. The transmitted linear frequency ramp with bandwidth $B$ centred around the carrier frequency $f_{\mathrm{c}}$ can be described as

$$
f_{\mathrm{T}}(t)=f_{\mathrm{c}}+\frac{B}{T_{\mathrm{c}}} t, \quad t \in\left[-\frac{T_{\mathrm{c}}}{2}, \frac{T_{\mathrm{c}}}{2}\right] .
$$

In [3] the intermediate frequency signal

$$
\begin{aligned}
s_{\mathrm{IF}}(t)= & \sum_{l=0}^{L-1} w(t) A \operatorname{rect}\left(\frac{t-l T_{\mathrm{r}}}{T_{\mathrm{c}}}\right) \\
& \cdot \mathrm{e}^{\left\{i 2 \pi\left[\frac{2 f_{\mathrm{c}}\left(R+v T_{\mathrm{r}} l\right)}{c}+\left(\frac{2 f_{\mathrm{c}} v}{c}+\frac{2 B\left(R+v T_{\mathrm{r}} l\right)}{c T_{\mathrm{c}}}\right) t\right]\right\}}
\end{aligned}
$$

is derived with a window function $w(t)$ and an arbitrary amplitude $A$. One single measurement consists of $L$ frequency

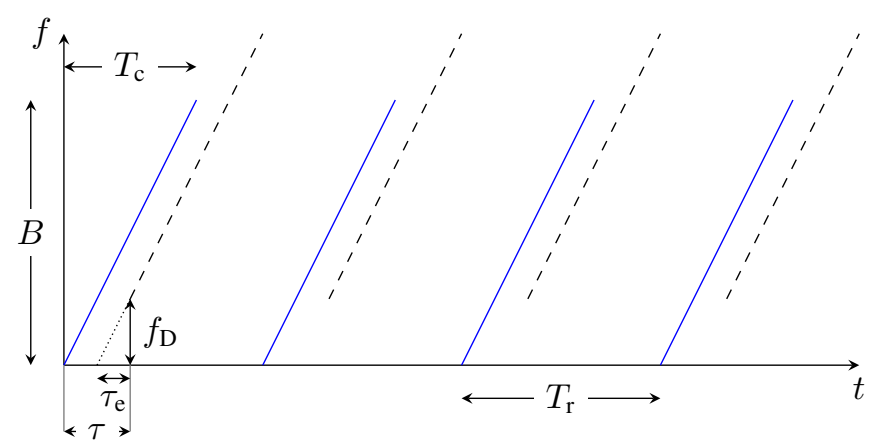

Fig. 1. Chirp-sequence modulation scheme: Four consecutive transmit chirps (-) and the received ramps (- - ). Due to the Doppler shift the ramps are shifted in frequency which can be expressed by a time error $\tau_{\mathrm{e}}$, which is usually neglected.

ramps which are repeated every $T_{\mathrm{r}}$. The movement of a target is assumed to be linear, thus the distance to the target for each ramp is set up with $R+v T_{\mathrm{r}} l$.

For signal processing the two-dimensional Fast Fourier Transform $(2 D-F F T)$ is applied to extract the range frequency $f_{\mathrm{R}}$ and velocity frequency of the intermediate signal $s_{\mathrm{IF}}(t)$

$$
f_{\mathrm{IF}}=\underbrace{\frac{2 f_{\mathrm{c}} v}{c}}_{=f_{\mathrm{D}}}+\underbrace{\frac{2 B R}{c T_{\mathrm{c}}}}_{=f_{\mathrm{R}}} \approx \frac{2 B R}{c T_{\mathrm{c}}}
$$

of each target. The first FFT is calculated using the samples of each ramp leading to a vector consisting of range cells with a peak at the target distance. The second calculated FFT for every range cell along the different ramps results in the Doppler frequency and therefore in the velocity of the target. The velocity resolution

$$
\Delta v=\frac{c}{2 f_{\mathrm{c}}} \Delta f_{\mathrm{v}}=\frac{c}{2 f_{\mathrm{c}} L T_{\mathrm{r}}}
$$

is determined for a given ramp repetition time $T_{\mathrm{r}}$ by the number of transmitted chirps. With the typically used approximation $\Delta f_{\mathrm{R}}=\frac{1}{T_{\mathrm{c}}}$ the range resolution is $\Delta R=\frac{c}{2 B}$.

The distance to the target is influenced by the Doppler frequency as in (3) and can be expressed as an error in the 


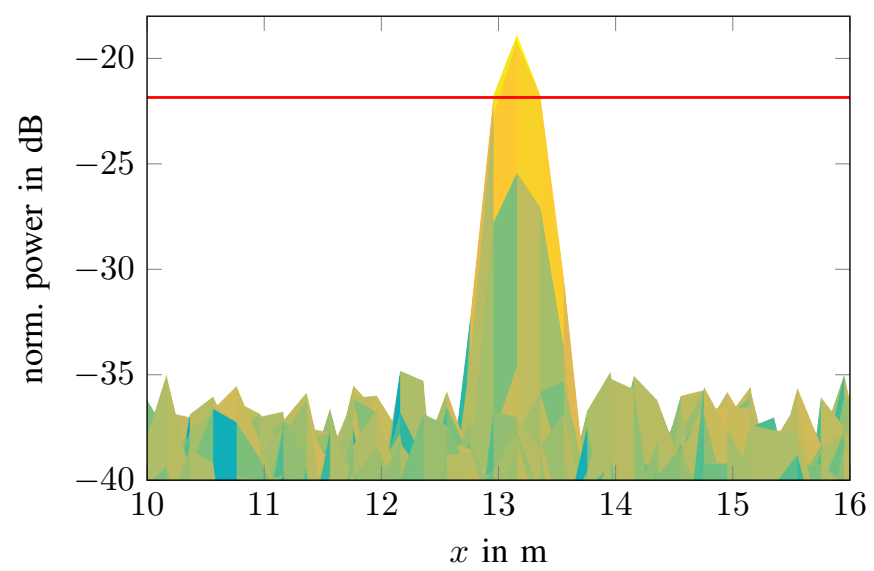

Fig. 2. Example of the peak in the 2D-FFT spectrum of a target at a distance of $12.5 \mathrm{~m}$ with a velocity of $55 \frac{\mathrm{m}}{\mathrm{s}}$. Due to the measurement time, the target migrates through different range cells resulting in a wide peak. The peak width is measured at the $3 \mathrm{~dB}$ threshold (—).

distance. The Doppler shift is then considered as a time error $\tau_{\mathrm{e}}$ as in Fig. 1 and expressed as a distance error

$$
R_{\mathrm{e}}=\frac{c}{2} \tau_{\mathrm{e}}=\frac{c}{2} \cdot \frac{T_{\mathrm{c}}}{B} \cdot f_{\mathrm{D}} \stackrel{(3)}{=} \frac{T_{\mathrm{c}} v f_{\mathrm{c}}}{B},
$$

which is usually small using a chirp-sequence modulation.

\section{RAnge Migration COMPEnsation}

As described in the previous section, the evaluation of the chirp-sequence modulation is a two-step process. To extract the velocity information, the $F F T$ is calculated along the different ramps for each range cell. Due to the target velocity the distance to it changes while the measurement takes place leading to a range cell migration. Increasing the bandwidth enhances the range resolution and hence decreasing the range cells leading to a stronger range cell migration effect. To ensure a better velocity resolution the number of transmitted ramps needs to be increased according to (4). This leads to a longer measurement time and with this to a stronger range cell migration. The disadvantageous effect of the range cell migration is a wider peak in the resulting $2 D-F F T$ spectrum which complicates the separation of two near targets.

In Fig. 2 a target is simulated at a distance of $12.5 \mathrm{~m}$ with a constant velocity of $55 \frac{\mathrm{m}}{\mathrm{s}}$. Using a chirp-sequence modulation the target travels $0.91 \mathrm{~m}$ while the measurement takes place and migrates through the approximately $0.20 \mathrm{~m}$ broad range cells. This leads to a broad peak in the range direction. As a quality factor the $3 \mathrm{~dB}$ peak width is used as shown in Fig. 2.

\section{A. Bandwidth Variation Modulation}

In [2] a modulation format is presented which prevents the range migration effect by altering the distance error $R_{\mathrm{e}}$ of (5). Therefore, the chirp bandwidth is changed for every transmitted ramp. The actual distance to the target for the $l$ th ramp is denoted by $R_{\mathrm{T}}^{(l)}$, the distance error with

$$
R_{\mathrm{e}}^{(l)}=\frac{T_{\mathrm{c}} v f_{\mathrm{c}}}{B},
$$

and the changing bandwidth of each chirp with $B^{(l)}$. The distance to the target can be written as

$$
R^{(l)}=R_{\mathrm{T}}^{(l)}+R_{\mathrm{e}}^{(l)} .
$$

To compensate the range migration effect

$$
\begin{aligned}
R^{(0)} & =R^{(l)} \\
R_{\mathrm{T}}^{(0)}+R_{\mathrm{e}}^{(0)} & =R_{\mathrm{T}}^{(l)}+R_{\mathrm{e}}^{(l)} \\
R_{0}+\frac{T_{\mathrm{c}} v f_{\mathrm{c}}}{B^{(0)}} & =R_{0}+v l T_{\mathrm{r}}+\frac{T_{\mathrm{c}} v f_{\mathrm{c}}}{B^{(l)}} \\
B^{(l)} & =\frac{B^{(0)} T_{\mathrm{c}} f_{\mathrm{c}}}{T_{\mathrm{c}} f_{\mathrm{c}}-B^{(0)} T_{\mathrm{r}} l}, \quad l \in[0, L-1]
\end{aligned}
$$

must be met. As already mentioned by the authors of [2], this condition is independent from the target velocity. By choosing a large bandwidth the frequency of the intermediate signal in (3) increases and a higher sampling frequency $f_{\mathrm{s}}$ is required.

\section{B. Chirp Duration Variation Modulation}

The variation of the bandwidth $B$ of the chirp ramps has the drawback that for the first ramps the bandwidth and hence the range resolution is low. To compensate the range migration effect the slope of the frequency ramps must be adapted. Instead of changing the bandwidth the chirp duration $T_{\mathrm{c}}$ of the ramps can be altered according to (10) leading to the condition

$$
T_{\mathrm{c}}^{(l)}=\frac{T_{\mathrm{c}}^{(0)} f_{\mathrm{c}}-B l T_{\mathrm{r}}}{f_{\mathrm{c}}}, \quad l \in[0, L-1],
$$

where $T_{\mathrm{c}}^{(l)}$ is the chirp duration of the $l$ th ramp. Fulfilling (12) leads to decreasing chirp durations while the time between each consecutive ramp $T_{\mathrm{r}}$ is unchanged to ensure a simple signal processing. If $T_{\mathrm{r}}$ would be adapted as well, a nonuniform sampling of the Doppler frequency must be applied.

By increasing the number of chirp ramps $l$ the chirp duration $T_{\mathrm{c}}^{(l)}$ for additional ramps decreases as well, but hardware requirements limit the minimal chirp duration at some point. Following (12), transmitting even more ramps would result in negative chirp durations. Looking at (1) negative times

$$
f_{\mathrm{T}}(t)=f_{\mathrm{c}}-\frac{B}{T_{\mathrm{c}}} t
$$

can be interpreted as falling ramps.

This modulation scheme is depicted in Fig. 3. The first ramps feature a rising ramp, while the last ramps are falling ones. In this illustration the fourth ramp would have a too short

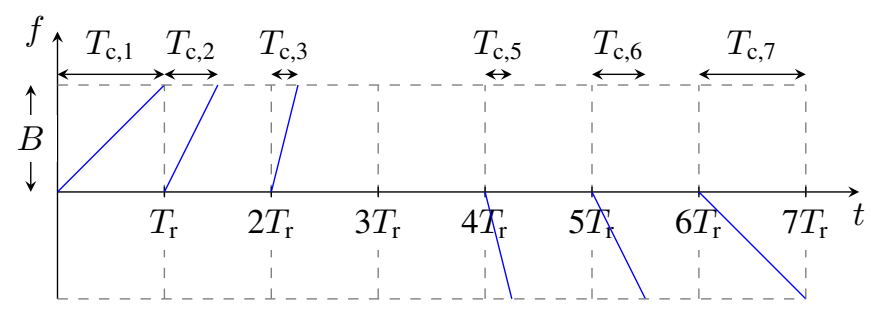

Fig. 3. Modulation scheme of the presented chirp duration variation modulation. 
duration to be realised and is therefore left out. This time slot with no transmitted signal is required to ensure a regular signal processing with uniform time steps for the Doppler frequency. If too many ramps are left out the side lobes of the window function in the Doppler spectrum rise which could lead to possible unwanted ghost targets.

\section{Simulation Results}

The variation of the bandwidth as well as the variation of the chirp duration are evaluated using a simulation environment. To guarantee a realistic simulation both phase noise and additive white Gaussian noise are used. The phase noise is modeled as described in [4] and implemented as in [5].

\section{A. Adapted Bandwidth Variation Modulation}

For the application in an automotive environment the specifications of the chirp radar need to be changed according to Section III-A and are listed in Table I. Fulfilling the condition (11) leads to a large range of bandwidth to be used.

Using a large number of ramps is ideal for an optimal velocity resolution. Also a large bandwidth will result in small range bins, in this example for the last ramps in a bin size of $0.07 \mathrm{~m}$. Due to the large variety of the used bandwidth and the large number of ramps the resulting peak is a bit enlarged. This is due to the fact that the bin size at the end is much smaller than at the beginning. To enhance the evaluation one could first evaluate the whole $2 D-F F T$ spectrum to extract the velocity information as in Fig. 4 (a). To extract the range information one could use the last ramps transmitted with the high bandwidth to ensure an optimal range resolution as depicted in Fig. 4 (b).

\section{B. Modulation Format Comparison}

To compare the proposed chirp duration variation modulation scheme to the chirp bandwidth variation and the standard approach with constant parameters the $3 \mathrm{~dB}$ peak width as introduced in Section III is used. To ensure comparability equal parameters are used. If the chirp duration is altered, the mean of the chirp duration is chosen such that it is equal to the other modulation formats. The parameters used for the comparison are listed in Table II.

To verify the range migration compensation a target with different constant velocities is simulated using the three modulation formats. The peak widths in range and velocity direction are listed in Table III. It can be clearly seen that the peak for the standard modulation format is increasing with higher velocities. For the bandwidth and chirp duration variation the

TABLE I

SPECIFICATIONS OF AdAPTED BANDWIDTh VARIATION MOdUlation

\begin{tabular}{lr} 
Parameter & Value \\
\hline carrier frequency $f_{\mathrm{c}}$ & $77 \mathrm{GHz}$ \\
bandwidth $B$ & $37-2260 \mathrm{MHz}$ \\
chirp duration $T_{\mathrm{c}}$ & $80 \mu \mathrm{s}$ \\
chirp repetition time $T_{\mathrm{r}}$ & $80 \mu \mathrm{s}$ \\
sampling frequency $f_{\mathrm{s}}$ & $20 \mathrm{MHz}$ \\
number of chirps $L$ & 2048
\end{tabular}

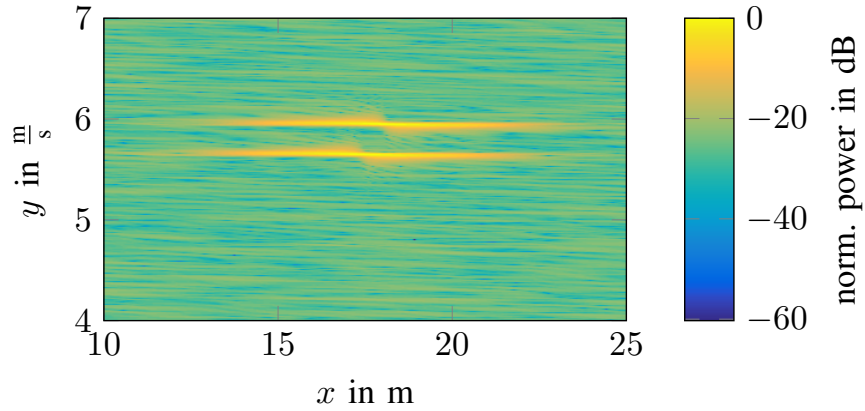

(a) Zoomed in $2 D-F F T$ spectrum using all ramps to extract the velocity.

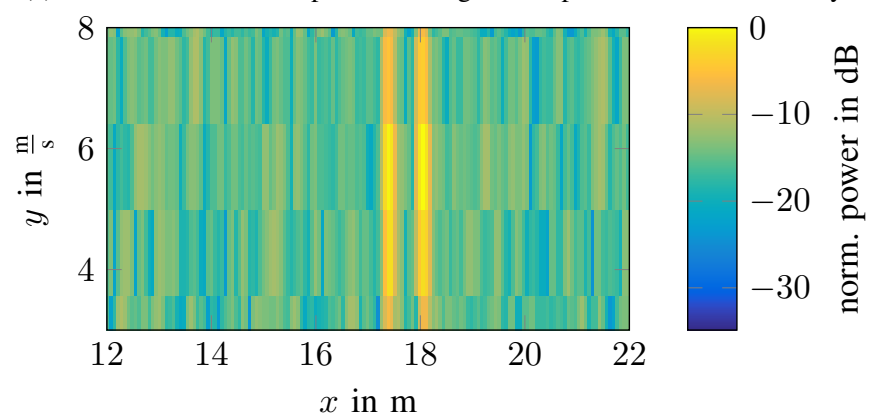

(b) Zoomed in $2 D-F F T$ spectrum using the last 16 ramps to extract the range information of the target. Narrow peaks in range direction but enlarged peaks in velocity direction.

Fig. 4. Evaluation of the adapted bandwidth variation using all ramps in (a) for the velocity and only the last ramps in (b) for the range information.

peak width is nearly constant for all velocities. Due to the compensation it is not dependent on the target velocity. The exact peak position inside a range or velocity cell has an influence on the calculated peak width. The two larger peak widths in the velocity direction of the chirp duration variation can be explained by such a behaviour. Using a small bandwidth at the first ramps results in larger range cells. This explains the larger peaks in the bandwidth variation than in the duration variation.

To separate two close targets the notch in the spectrum between them must be lower than $3 \mathrm{~dB}$. To show the advantage of the proposed modulation format two close targets at a distance of $12.5 \mathrm{~m}$ and $13 \mathrm{~m}$ are simulated. With the standard modulation format the two peaks can not be distinguished as can be seen in Fig. 5 (a). Using a changing bandwidth the notch appears but does not fulfill the required $3 \mathrm{~dB}$, see Fig. 5 (b). Only with the chirp duration variation the condition can be fulfilled as in Fig. 5 (c).

TABLE II

SPECIFICATIONS FOR THE COMPARISON

\begin{tabular}{lrrr} 
Parameter & Standard & Bandwidth Variation & Duration Variation \\
\hline$f_{\mathrm{c}}$ & $77 \mathrm{GHz}$ & $77 \mathrm{GHz}$ & $77 \mathrm{GHz}$ \\
$B$ & $750 \mathrm{MHz}$ & $181.5-5406 \mathrm{MHz}$ & $750 \mathrm{MHz}$ \\
$T_{\mathrm{c}}$ & $40 \mu \mathrm{s}$ & $40 \mu \mathrm{s}$ & $80-10.4 \mu \mathrm{s}$ \\
$T_{\mathrm{r}}$ & $80 \mu \mathrm{s}$ & $80 \mu \mathrm{s}$ & $80 \mu \mathrm{s}$ \\
$f_{\mathrm{s}}$ & $10 \mathrm{MHz}$ & $100 \mathrm{MHz}$ & $35 \mathrm{MHz}$ \\
$L$ & 206 & 206 & 206
\end{tabular}


TABLE III

PEAK WIDTH COMPARISON

\begin{tabular}{ccccccc} 
Velocity in $\frac{\mathrm{m}}{\mathrm{s}}$ & \multicolumn{2}{c}{ Standard } & \multicolumn{3}{c}{ Bandwidth Var. } & \multicolumn{2}{c}{ Duration Var. } \\
& $\begin{array}{c}\text { in } R \\
\text { in } \mathrm{m}\end{array}$ & $\begin{array}{c}\text { in } v \\
\text { in } \frac{\mathrm{m}}{\mathrm{s}}\end{array}$ & $\begin{array}{l}\text { in } R \\
\text { in } \mathrm{m}\end{array}$ & $\begin{array}{l}\text { in } v \\
\text { in } \frac{\mathrm{m}}{\mathrm{s}}\end{array}$ & $\begin{array}{l}\text { in } R \\
\text { in } \mathrm{m}\end{array}$ & $\begin{array}{l}\text { in } v \\
\text { in } \frac{\mathrm{m}}{\mathrm{s}}\end{array}$ \\
\hline 0 & 0.42 & 0.24 & 0.49 & 0.22 & 0.40 & 0.20 \\
15 & 0.42 & 0.25 & 0.49 & 0.22 & 0.44 & 0.22 \\
25 & 0.44 & 0.27 & 0.48 & 0.24 & 0.40 & 0.28 \\
35 & 0.48 & 0.29 & 0.48 & 0.24 & 0.44 & 0.19 \\
45 & 0.52 & 0.30 & 0.48 & 0.22 & 0.40 & 0.27 \\
55 & 0.60 & 0.34 & 0.48 & 0.24 & 0.42 & 0.18
\end{tabular}

\section{Noise Consideration}

In Section IV-A the last 16 ramps are used to extract the range information. A larger number of ramps could be used to increase the signal-to-noise ratio $(S N R)$. It is best to use only ramps with a nearly constant bandwidth.

In Section IV-B the parameters are chosen such that the average noise power level is equal. Using the standard chirpsequence modulation the power of a target is distributed over several range cells depending on its velocity in contrast to the bandwidth variation. This results in a higher target peak as can be seen in Fig. 5 (b) in comparison to (a). For the duration variation each consecutive chirp is shorter and with a large number of chirps even some chirps are left out. For the presented parameters this results in $50 \%$ less samples and thus in a $3 \mathrm{~dB}$ higher average noise power.

\section{CONCLUSION}

The introduced chirp duration variation modulation is able to cope better with the range migration effect than the standard chirp-sequence procedure and the bandwidth variation modulation. To evaluate the results the $3 \mathrm{~dB}$ peak width is defined. It is shown that for a target with a velocity of $55 \frac{\mathrm{m}}{\mathrm{s}}$ the proposed method results in $30 \%$ and $47 \%$ smaller peak widths for the range and the velocity direction compared to the standard approach.

\section{REFERENCES}

[1] E. Schubert, F. Meinl, M. Kunert, and W. Menzel, "High Resolution Automotive Radar Measurements of Vulnerable Road Users - Pedestrians \& Cyclists," in IEEE MTT-S International Conference on Microwaves for Intelligent Mobility (ICMIM), Apr. 2015, pp. 1-4.

[2] T. Nagano, T. Iwamoto, T. Hara, and Y. Takeda, "Range Migration Compensation for Moving Targets with Unknown Constant Velocity in Chirp Radars," in Proceedings of the 8th European Radar Conference, Oct 2011, pp. 125-128.

[3] V. Winkler, "Range Doppler Detection for automotive FMCW Radars," in Proceedings of the 4th European Radar Conference (EuRAD), Oct. 2007, pp. 166-169.

[4] A. Demir, A. Mehrotra, and J. Roychowdhury, "Phase Noise in Oscillators: A Unifying Theory and Numerical Methods for Characterization," IEEE Transactions on Circuits and Systems I: Fundamental Theory and Applications, vol. 47, no. 5, pp. 655-674, May 2000.

[5] A. Frischen, J. Hach, and C. Waldschmidt, "Performance Degradation in Cooperative Radar Sensor Systems due to Uncorrelated Phase Noise," in Proceedings of the 11th European Radar Conference (EuRAD), Oct. 2014, pp. 241-244.

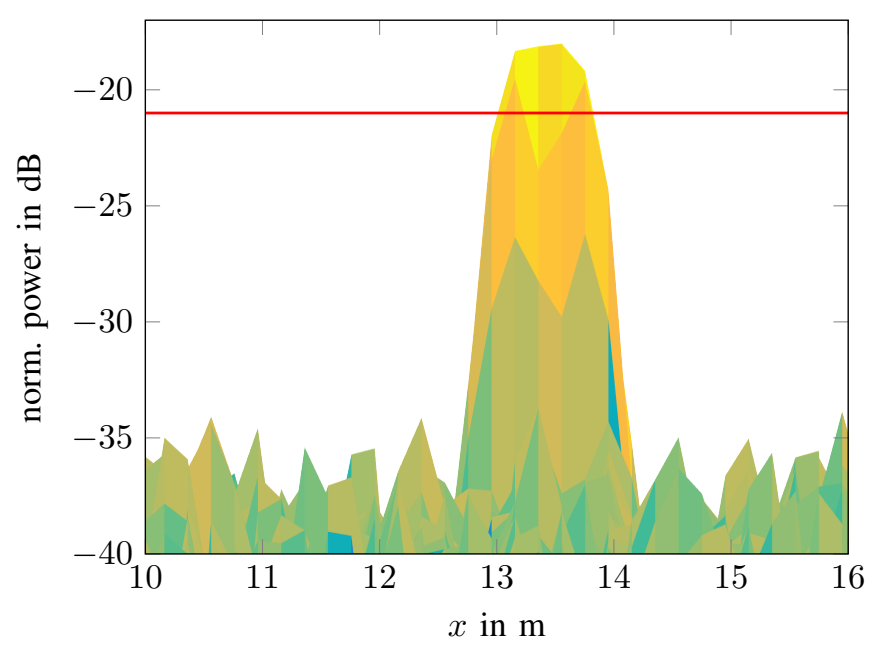

(a) Standard chirp-sequence approach resulting in two non-separable peaks.

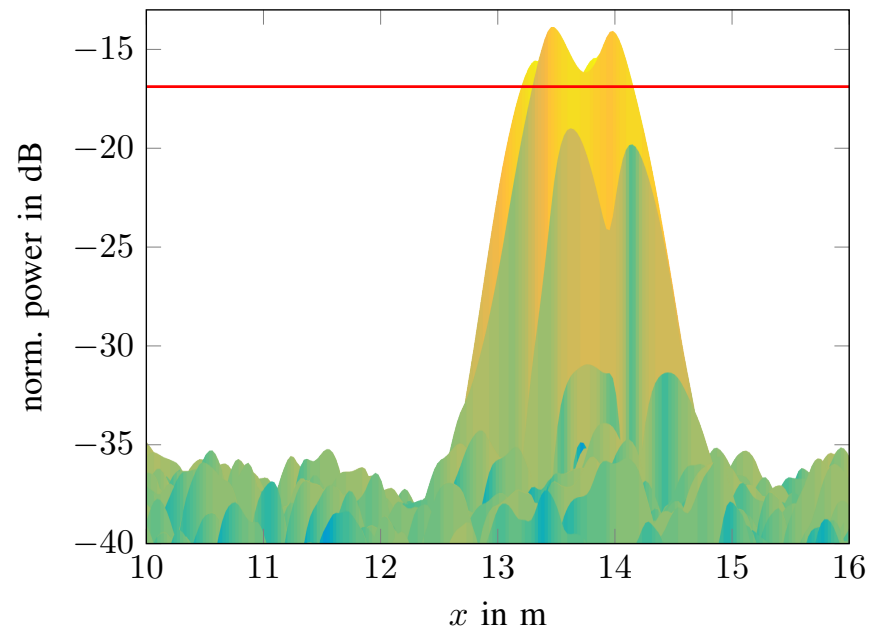

(b) Using the bandwidth variation the notch between the peaks appears compared to the standard approach, but the targets are still not separable.

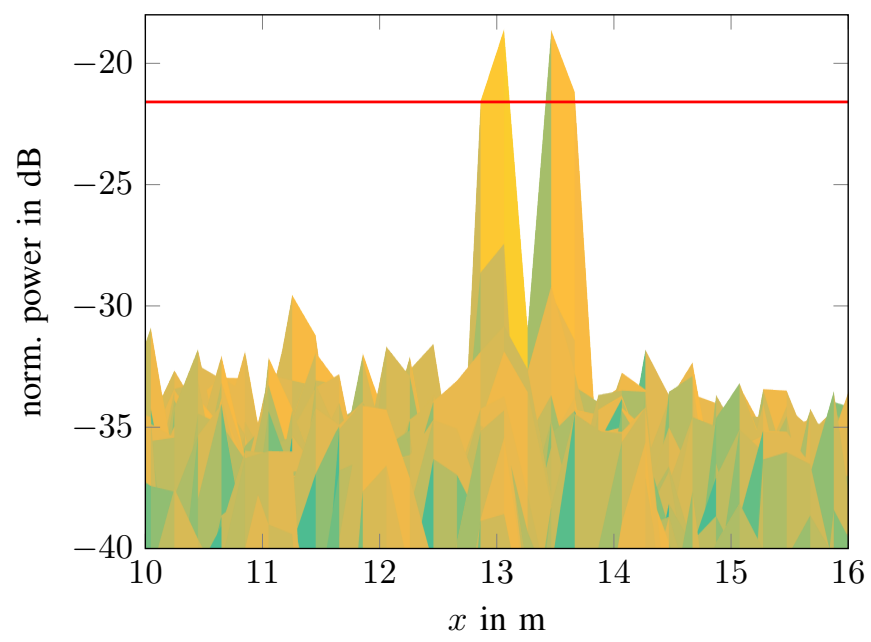

(c) Only with the chirp duration variation the peaks can be distinguished.

Fig. 5. Comparison of the accuracy of different modulation schemes for two close targets with $55 \frac{\mathrm{m}}{\mathrm{s}}$ at $12.5 \mathrm{~m}$ and $13 \mathrm{~m}$, respectively. In (a) for the standard modulation, in (b) for the bandwidth, and in (c) for the chirp duration variation modulation. 